\title{
Round Cell Count
}

National Cancer Institute

\section{Source}

National Cancer Institute. Round Cell Count. NCI Thesaurus. Code C142288.

The determination of the amount of round shaped cells, mainly comprised of white blood cells and immature spermatogenic cells, present in a sample. 